\title{
Terrain Matching Based on Adaptive Digital Elevation Map
}

\author{
Bin Yan ${ }^{1}$, Yiming Wang ${ }^{1}$, Lun Feng ${ }^{2}$, Hui Zhou ${ }^{2}$ and Zefeng Jiang ${ }^{2}$ \\ ${ }^{1}$ University of Electronic Science and Technology of China, Chengdu, China \\ ${ }^{2}$ State Grid Sichuan Electric Power Company, Chengdu, China \\ *Corresponding author
}

\begin{abstract}
In order to improve the navigation precision of the classical sandia inertial terrain aided navigation (SITAN), replacing kalman filter (KF) with particle filter (PF) for nonlinear system, this paper modifies and compensates inertial navigation system (INS) by terrain matching. Moreover, this paper proposes an adapti ve scaling method to improve the digital elevation map (DEM)'s precision of the region with distinct topographic features, and then improves the navigation precision.
\end{abstract}

Keywords-SITAN; PF; scaling down; INS

\section{INTRODUCTION}

Harsh environment, such as high altitude and unmanned area, will be encountered frequently while inspecting the transmission lines with small rotor unmanned aerial vehicle(UA V).INS cannot guarantee the normal work of UA V under the serious absence of satellite signals, even causing the UA V to fall. Passive navigation, such as visual navigation [1-2] and geomagnetic navigation [3-4], is often used with INS to solve UAV's navigation in signal barrier area. Considering that the inspection of transmission lines is often carried out in mountainous areas, terrain matching is the best method to solve signal barrier.

The factors that affect terrain aided navigation(TAN)'s accuracy are:(1) INS;(2)DEM;(3)Features of terrain;(4) algorithm;(5)altitude measurement. This paper proposes an SITAN algorithm based on adaptive scale DEM. In the steep terrain features area, scaling down the DEM with bilinear interpolation based on wavelet transform is used to improve the DEM's precision and then improve the accuracy of navigation.

\section{ST ATUS OF THE RESEARCH}

TAN [5-6] is a combined navigation technique, which compensates the accumulated error of inertial navigation using terrain and radar altimeter information. In recent years, many scholars have optimized the terrain contour matching (TERCOM), SITAN [7] and TERPROM [8] three classical TAN algorithms.

In [9], Jiang Wu used an improved multipath terrain contour matching algorithm to deal with the terrain elevation data provided by laser scanning radar to improve the flexibility and timeliness of terrain matching. With a deep analysis and comparison, the paper [10] demonstrated the performance of unscented kalman filter (UKF) is better than that of extended kalman filter (EKF), but EKF is more feasible in practical application. In [11], the BP neural network was applied to the terrain linearization and EKF, in which the performance was superior to traditional SITAN. By adding PF [12-13], the problem of nonlinear and non-Gaussian was solved, and the results of terrain matching under different parameter were compared. The new acquisition mode adopted the TERCOM to implement the uniform voting algorithm, which could obtain the initial position more quickly [14]. Burak Turan [15] further demonstrated the ability of the sequential importance sampling (SIS-R) and sampling importance resampling (SIR) to deal with nonlinearity and the probability distributions of different types and even multimode, and SIS-R achieved a higher precision by less computation. The speed of searching all possible path values was accelerated by transforming the terrain elevation data into the digital attribute database [16] Combining with EKF, terrain extremum correlation was used to improve the accuracy and real-time [17]. Orhan eroglu [18] proposed a unique scoring algorithm based on original algorithm, and in the search process, the large search space became linear solvable small space, which improved search speed and reduced search time.

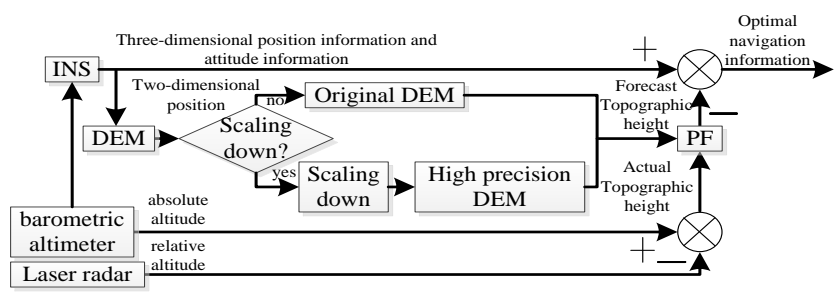

FIGURE I. TERRAIN MATCHING PRINCIPLE

\section{TERRAIN MATCHING BASED ON ADAPTIVE SCALING FOR DEM}

With reference information providing by the existing DEM, TAN system [19] makes UAV in the absence of satellite signals calculating the current navigation information accurately by correcting INS accumulation error in auxiliary. The precision of DEM, mostly in the $30 \sim 50 \mathrm{~m}$, is one of the decisive factors for the navigation accuracy, and the higher precision DEM occupies more me mory. Considering the small UAV's load and the real-time performance, we propose a SITAN based on the adaptive scaling of DEM. The terrain characteristic area is scaled down through a bilinear interpolation method based on wavelet transform according to 
the current terrain steepness to improve precision, and solve the exponential data of the high precision DEM.

\section{A. SITAN}

SITAN began in the 1970s.UA V's altitude information h_a is calculated with the barometric altimeter data and INS height information. Terrain height $\mathrm{h} \_\mathrm{m}$ under current position is estimated through the two-dimension position (L, $\lambda$ ) of UAV provided by inertial navigation.

The measurement of the system, prediction information of UA V relative altitude $\hat{h} \hat{r}$ which is the difference between $h \_a$ and $h \_m$ is used to establish a nonlinear measurement equation with actual altitude of $\mathrm{UAV} \tilde{\mathrm{h}} \tilde{r}_{\mathrm{r}}$ measured by radar altimeter. Combining measurement equation with error equation of INS, error value can be estimated by KF to compensate and correct the navigation information obtained by inertial navigation.

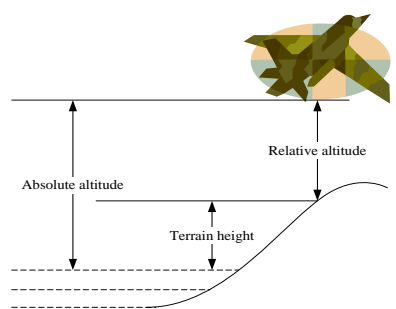

\section{FIGURE II. THE PRINCIPLE PF TERRAIN HEIGHT MATCHING}

Because of compensating error by linear KF in the classical SITAN, the terrain must be linearized, which leads to lower navigation accuracy. PF is one of the powerful tools to solve this kind of nonlinear problem. A group of random samples that called particles is produced in the state space, the weight value and position of each particle are adjusted according to the observation information, and then the empirical condition distribution of the original particles is corrected.

1) Initialization: collection of particles from the initial distribution $\mathrm{p}\left(\mathrm{x} \_0\right), \mathrm{x} \_0^{\wedge}((\mathrm{i})) \sim \mathrm{p}\left(\mathrm{x} \_0\right), \omega \_0^{\wedge}((\mathrm{i}))=1 / \mathrm{N}$;

2) Process oftransfer:

systemmodel is as follows.

$$
\begin{aligned}
& x(t)=F(t) x(t)+G(t) w(t) \\
& y=h \_m(p-x(1: 2))+e
\end{aligned}
$$

Among the equation, $\mathrm{x}=\left[\delta \mathrm{L} \delta \lambda \delta \mathrm{V}_{-} \mathrm{E} \delta \mathrm{V} \_\mathrm{N} \emptyset_{-} \mathrm{E} \emptyset \_\mathrm{N}\right.$ $\emptyset \_U \nabla \_$ax $\nabla \_$ay $\xi \_$bx $\xi \_$by $\left.\xi \_b z\right]^{\wedge} \mathrm{T}, \mathrm{w}=\left[\omega \_\right.$ax $\omega \_$ay $\omega \_$gx $\left.\omega \_g x \omega \_g x\right]^{\wedge} T$, State transition matrix $F(t)$ and Noise driven matrix $G(t)$ is achieved by Inertial navigation error equation, $\mathrm{p}^{\wedge}=\left[\mathrm{L}^{\wedge} \lambda\right]^{\wedge} \mathrm{T}$ is two-dimensional horizontal position calculated by INS.

According to Bayesian theory, the problem of state estimation is to calculate the confidence $\mathrm{p}\left(\mathrm{x}_{-} \mathrm{t} \mid \mathrm{y}_{-}(1: \mathrm{t})\right)$ of current state $X(t)$ based on previous series of prior knowledge recursion, and it needs forecast and update two steps.

a) Prediction: use the system model to predict the prior probability density of state, assume that the system state transfer obeys the first order Markov model,y(t) is only relevant to the current state $\mathrm{x}(\mathrm{t}): \mathrm{p}\left(\mathrm{x} \_\mathrm{t} \mid \mathrm{y}_{-}(1: \mathrm{t}-1)\right)=\int \mathrm{p}\left(\mathrm{x} \_\mathrm{t}\right.$ $\left.\mid \mathrm{x} \_(\mathrm{t}-1)\right) \mathrm{p}\left(\mathrm{x} \_(\mathrm{t}-1) \mid \mathrm{y}_{-}(1: \mathrm{t}-1)\right) \mathrm{d} \mathrm{x}_{-}(\mathrm{t}-1)$, the state transfer PDF $\mathrm{p}\left(\mathrm{x} \_\mathrm{t} \mid \mathrm{x} \_(\mathrm{t}-1)\right)$ is available by the equation of state. b) Updating: the prior probability density is modified by the latest measurement value, and the posterior probability density is obtained, which is to revise the previous estimate.p(x_t $\left.\mid y_{-}(1: t-1)\right)=p\left(y \_t \mid x_{-} t\right) p\left(x \_t \mid y \_(1: t-1)\right) / p\left(y \_t\right.$ $\left.\mid y_{-}(1: t-1)\right)$. The likelihood PDF $p\left(y_{-} t-x_{-} t\right)$ is derived from the measurement equation. Introducing Monte Carlo to solve High-dimensional integral;

3) Decision stage

By [20], it is known that sampling is easier when the importance function $\mathrm{q}$ is $\mathrm{q}\left(\mathrm{x}_{-} \mathrm{t} \mid \mathrm{x} \_(\mathrm{t}-1), \mathrm{y}_{-} \mathrm{t}=\mathrm{p}\left(\mathrm{x} \_\mathrm{t} \mid \mathrm{x} \_(\mathrm{t}-1), \mathrm{y}_{-} \mathrm{t}\right)\right.$, The corresponding weight is $\omega_{-} \mathrm{t}^{\wedge}((\mathrm{i}))=\tilde{\omega}_{-}(\mathrm{t}-1)^{\wedge}((\mathrm{i})) \mathrm{p}\left(\mathrm{y} \_\mathrm{t}\right.$ $\left.\mid \mathrm{x}_{-} \mathrm{t}^{\wedge}((\mathrm{i}))\right), \quad$ normalized, i.e. $\quad \tilde{\omega}_{-} \mathrm{t}^{\wedge}((\mathrm{i}))=\omega_{-} \mathrm{t}^{\wedge}((\mathrm{i})) / \bar{\Sigma}$ $\left.-\overline{\mathrm{j}}=1)^{\wedge} \mathrm{N} \omega{ }_{-} \mathrm{t}^{\wedge}(\mathrm{j})\right)$

4) Resampling stage

For the "particle degeneration" phenomenon caused by continuous iterative computation of importance sampling, the number of effective particles after each decision is calculated, $\mathrm{N}^{\wedge}$ eff $\approx 1 / \sum 1^{\wedge} \mathrm{N}\left(\omega_{-} \mathrm{t}^{\wedge}((\mathrm{i}))\right)^{\wedge} 2$. If the number of effective particles is less than the set threshold, the particle $\operatorname{set}\left\{\tilde{\mathrm{x}} \mathbf{t}^{\wedge}((\mathrm{i})), \omega_{-} \mathrm{t}^{\wedge}((\mathrm{i}))\right\}$ is resampling according to the weight proportion of particles to produce a new particle set $\left\{\mathrm{x} \_\mathrm{t}^{\wedge}((\mathrm{i})), 1 / \mathrm{N}\right\}$;

5) output

Calculate the state estimate for the t-time: $\mathrm{x}_{-}^{\wedge} \mathrm{t}=\sum_{-}(\mathrm{i}=1)^{\wedge} \mathrm{N}$ $\tilde{x_{-}} t^{\wedge}((i)) \tilde{\omega} t^{\wedge}((i))$.

The velocity and position information obtained by inertial navigation are compensated by this state value, and the final navigation information is obtained.

\section{B. Adaptive DEM Scaling Down}

Because the prediction information is calculated by the difference between the absolute height information of INS and the relative altitude obtained by DEM, the accuracy of DEM is the decisive factor of final navigation. In order to avoid the large amount of data brought by high precision data which is difficult to obtain, this paper proposes whether the map scale would be pushed to the high precision map according to the terrain feature to improve the accuracy of navigation system

The average terrain slope $\mathrm{K}$ of the proposed patrol area is calculated by the software ArcGIS, and the current position topographic slope $\mathrm{k} \_\mathrm{y}=\left(\mathrm{h} \_\mathrm{t}-\mathrm{h} \_(\mathrm{t}-1)\right) /\left(\mathrm{y} \_\mathrm{t}-\mathrm{y} \_(\mathrm{t}-1)\right), \mathrm{h}$ is the absolute height in the body coordinate system, and $y$ is the position of the head direction. High precision DEM are obtained through super-resolution technology while k_y $>\mathrm{k}$.

Super-resolution technology, without changing the image detection system, is to use the existing low-resolution (LR) image to obtain a high-resolution (HR) image. Because of the particularity of DEM, the super-resolution method based on interpolation is adopted. Yingyue Sun [21] proposed a superresolution reconstruction method of wavelet local adaptive interpolation to preserve the details of the original image better Hao Yu [22] combined the square root model method with the wavelet multi-scale analysis method, which made the terrain surface detail smoother. In order to ensure the real-time flight, this paper chooses bilinear interpolation method, at the same time to solve the phenomenon of ambiguity and sawtooth appearing at the edge, introduces wavelet transform, a kind of 
layered sub band transform, to make a linear operation with information retention.

1) Bilinear interpolation method: Bilinear interpolation, that is using the Q_11,Q_12,Q_21,Q_22 of 4 adjacent points around $\mathrm{P}(\mathrm{x}, \mathrm{y})$ to make a linear interpolation in the $\mathrm{x}, \mathrm{y}$ direction.

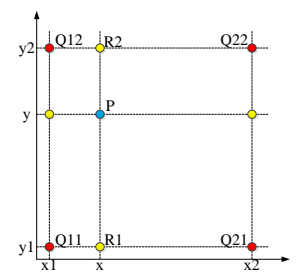

FIGURE III. BILINEAR INTERPOLATION METHOD

a) Linear interpolation of Q_11 and Q_21 in $\mathrm{x}$ direction to obtain $\mathrm{R} \_1$ value: $\mathrm{f}\left(\mathrm{R} \_1\right) \approx\left(\mathrm{x} \_2-\mathrm{x}\right) /\left(\mathrm{x} \_2-\mathrm{x} \_1\right) \mathrm{f}\left(\mathrm{Q} \_11\right)+(\mathrm{x}-$ X_1)/(x_2-x_1 ) f(Q_21 )

b) Linear interpolation of $\mathrm{Q} \_12$ and Q_22 in $\mathrm{x}$ direction to obtain $\mathrm{R} \_2$ value: $\mathrm{f}\left(\mathrm{R} \_2\right) \approx\left(\mathrm{x} \_2-\mathrm{x}\right) /\left(\mathrm{x} \_2-\mathrm{x} \_1\right) \mathrm{f}\left(\mathrm{Q} \_12\right)+(\mathrm{x}-$ $\left.X \_1\right) /\left(x \_2-x \_1\right) f\left(Q \_22\right)$

c) The $\mathrm{R} \_1$ and $\mathrm{R} \_2$ are linearly interpolated in the $\mathrm{Y}$ direction, and the value of $\mathrm{P}$ is obtained: $\mathrm{f}(\mathrm{P}) \approx\left(\mathrm{y} \_2-\mathrm{y}\right) /\left(\mathrm{y}_{-} 2-\right.$ y_1 ) $\mathrm{f}\left(\mathrm{R} \_1\right)+\left(\mathrm{y}-\mathrm{y} \_1\right) /\left(\mathrm{y} \_2-\mathrm{y} \_1\right) \mathrm{f}\left(\mathrm{R} \_2\right)$

2) Bilinear interpolation method based on wavelet: Wavelet transform can decompose the DEM information into different spatial resolution, frequency characteristics and directional characteristics of the sub band signal, while processing low-frequency long and high-frequency short time features, so bilinear interpolation based on wavelet transform can better maintain the rich high-frequency components. For the real function $g(x) \in L^{\wedge} 2(R) \quad\left(L^{\wedge} 2(R)\right.$ is in the square Integrable real space), if $\mathrm{G}(\omega)$, the Fourier transform of $\mathrm{g}(\mathrm{x})$,satisfies: $\mathrm{Cg}=\int_{-} \mathrm{g}^{\wedge}\left(|\mathrm{G}(\omega)|^{\wedge} 2 /|\omega|\right) \mathrm{d} \omega<\infty, \mathrm{g}(\mathrm{x})$ is called a basic wavelet, and it is translated and scaled to obtain a small wave sequence : g_(a,b) $(x)=|a|^{\wedge}(1 / 2)^{*} g((x-b) / a)$. a, scaling parameters, determines the width of the wavelet base function. $\mathrm{b}$,translation parameters ,determines the wavelet function along the $x$-axis translation position. For any $f(x)$ based on wavelet $g(x)$, the wavelet transform is the inner product of $f(x)$ and g_(a,b) (x): W $\{\mathrm{f}(\mathrm{x})\}=\mathrm{W} \_\mathrm{f}(\mathrm{a}, \mathrm{b})=\left\langle\mathrm{f}, \mathrm{g} \_(\mathrm{a}, \mathrm{b})\right\rangle=1 / \sqrt{ }(|\mathrm{a}|) \cdot \int_{-} \mathrm{g}^{\wedge}$ $f(x) \cdot g((x-b) / a) d x$.

The refactoring formula is $: f(x)=1 / C \_g \quad \int_{-}\left(R^{\wedge}\right)^{\wedge} \int_{-} R^{\wedge} 1 / a^{\wedge} 2$ $<$ f,g_(a,b) $>$ g_(a,b) (x)dadb

In two-dimensional discrete wavelet transform, firstly, the elevation data of each row of the DEM is transformed by a wavelet, the average and detail coefficients of each line elevation data are generated, and then a wavelet is used to transform the row-transformed column to produce the average and detail coefficients of the whole data elevation map.

The concrete steps of the DEM scale are as follows:

a) The DEM is decomposed into low-frequency and high-frequency in different directions by wavelet transform. b) The high-frequency part is processed by bilinear interpolation method, and the corresponding high-frequency part is constructed.

c) The original DEM and the high frequency data processed by bilinear interpolation method is used as low and high frequency part in the inverse wavelet transform, and then the DEM data corresponding to magnification is obtained.

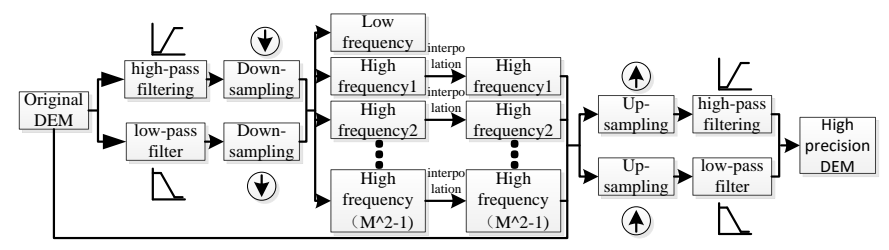

FIGURE IV. BILINEAR INTERPOLATION METHOD BASED ON WAVELET TRANFORM

\section{IV.SIMULATION RESULT}

A small four-rotor UAV was used to fly in a region of Sichuan to obtain the actual flight trajectory and collect threeaxis gyroscope and acceleration data. DEM obtained from Chinese Scientific Data Service platform is transformed to txt document by GLOBALMAPPER, and then imports it into $\mathrm{m}$ function to get the terrain height at any position. Because the test condition is limited, this paper simulates under MATLAB only. The specific program flow chart is as follows:

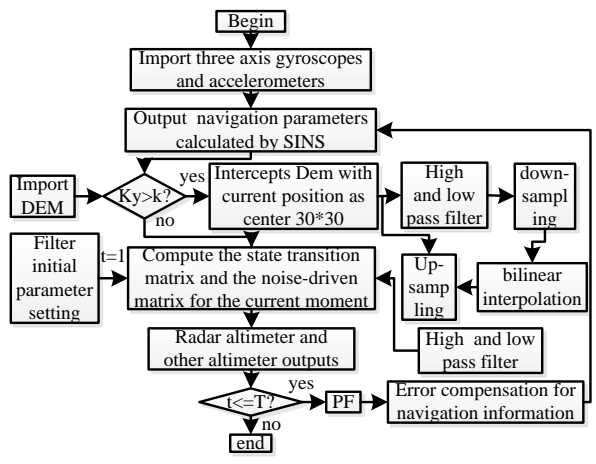

FIGURE V. ALGORITHM FLOW CHART ILINEAR

A DEM of n32e97 in Sichuan Province was obtained by the Chinese Scientific Data Service platform, processing which by using bilinear interpolation method on the MATLAB platform, and the simulation results are as follows:
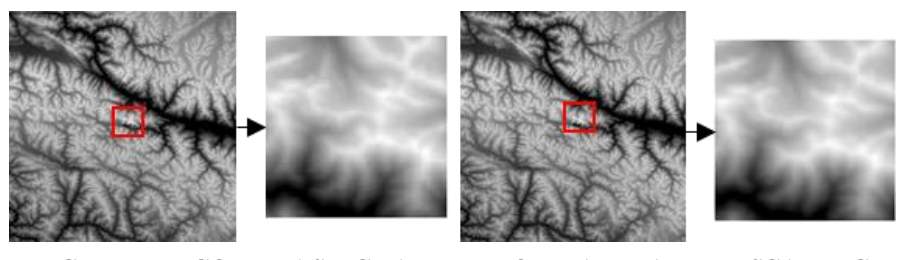

FIGURE VI. CONTRAST CHART BEFORE AND AFTER SCALING

The single bilinear interpolation method can improve the resolution of DEM, but there are some defects in the degradation of detail information after processing. Therefore, wavelet transform is introduced, the Harr wavelet is used as the 
wavelet base function, the same DEM is used as simulation data, and bilinear interpolation method based the wavelet is applied to improve the loss of high-frequency information.

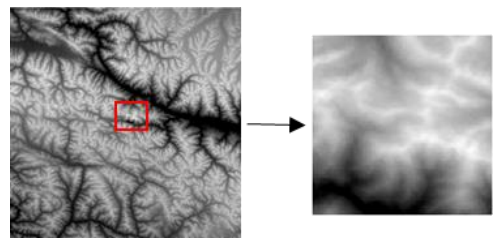

FIGURE VII. DEM AFTER ADDING WAVELET TRANSFORM

According to the electric parameters when UAV beg in to work and corresponding error parameter of the sensor model used in the system, the initial state and the state distribution are set up. The SITAN based on PF and the SITAN based on adaptive elevation map are used respectively to simulate while setting the number of particles to 20000.The obtained navigation information is subtracted from the threedimensional position information obtained from the actual flight trajectory, and the final error curve obtained is as follows:
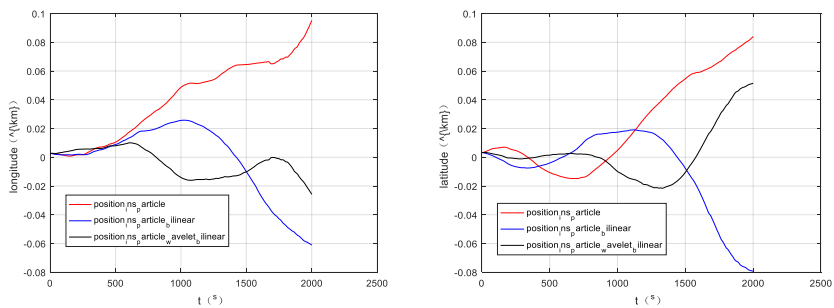

FIGURE VIII. COMPARISON OF ERRORS

In all the algorithms used in this paper, the SITAN based on $\mathrm{PF}$ is adopted, and it can be seen from the above diagram that better error compensation effect can be obtained because of the high resolution DEM after adaptive scale .Calculate its mean error value respectively, and show it as follows:

TABLE I. T ABLE TYPE ST YLES

\begin{tabular}{|l|c|c|c|}
\hline $\begin{array}{c}\text { Algori thm } \\
\text { Direction }\end{array}$ & $\begin{array}{c}\text { SITAN Based } \\
\text { On PF }\end{array}$ & $\begin{array}{c}\text { Add bilinear } \\
\text { interpolation }\end{array}$ & $\begin{array}{c}\text { Adding wavelet } \\
\text { transform }\end{array}$ \\
\hline longitude $(\mathrm{m})$ & 35.1 & 19.3 & 8.6 \\
\hline latitude $(\mathrm{m})$ & 28.6 & 19.4 & 12.1 \\
\hline
\end{tabular}

At the same time, it can be seen from the simulation diagram that the particle will degenerate and the filter result divergence. Therefore, the improved PF algorithm can be used to further improve the accuracy of terrain matching later.

\section{CONCLUSION}

Based on the classical SITAN, this paper designs a TAN algorithm based on $\mathrm{PF}$, improves the overall navigation accuracy of the system by better adaptability to the nonlinear system, and on this basis, designs the DEM based on the terrain fluctuation feature for adaptive scaling. Compared with the original terrain matching algorithm, it can improve the navigation accuracy of the terrain, and solve the problem of signal barrier in the transmission line patrol in mountainous area.

\section{REFERENCES}

[1] Lu H, Zhang F, Wu J. A new real time environment perception method based on visual image for micro UAS flight control[C]// Guidance, Navigation and Control Conference. IEEE, 2014:2515-2519.

[2] Liu X C, Wang H, Fu D, et al. An area-based position and attitude estimation for unmanned aerial vehicle navigation[J]. SCIENTIA SINICA Technologica, 2015, 58(5):916-926.

[3] Hua M U, Ren Z X, Xiao-Ping H U, et al. Information fusion strategy and performance for marine Inertial/Geomagnetic navigation system[J]. Journal of Chinese Inertial Technology, 2007.

[4] Liu F, Zhou X G, Yang Y, et al. Geomagnetic matching location using correlative method[J]. Journal of Chinese Inertial T echnology, 2007.

[5] Carreno S, Wilson P, Ridao P, et al. A survey on Terrain Based Navigation for AUVs[C]// Oceans. 2010:1-7.

[6] Johnson A E, Mont gomery J F. Overview of Terrain Relative Navigation Approaches for Precise Lunar Landing[C]// Aerospace Conference. IEEE Xplore, 2008:1-10.

[7] Xude Liu, Terrain aided navigation system technology [M]. Publishing House of Electronics Industry, 1994

[8] Cowie M, Wilkinson N, Powlesland R. Latest development of the TERPROM $®$ Digital Terrain System (DT S) [C]// Position, Location and Navigation Symposium, 2008 IEEE/ION. 2008:1219 - 1229.

[9] Wu J, Fei W, Li Q. A novel location method based on 2D laser scanning sensor terrain matching for UAV autonomous flight[C]// Iet International Conference on Information Science and Control Engineering. IET, 2014:3.78-3.78.

[10] H. Mei, Y. Jing, L. Qin, Z. Shuangshuang. EKF and UKF algorithms and the application to terrain-aided navigation, Chinese Journal of Scientific Instrument, 2008, 29(4): pp. 438-443

[11] Liu C, Wang H, Yao P. On terrain-aided navigation for unmanned aerial vehicle using B-spline neural network and extended Kalman filter[C]// IEEE Chinese Guidance, Navigation and Control Conference. IEEE, 2014:2258-2263.

[12] Melo J, Matos A. On the use of particle filters for terrain based navigation of sensor-limited AUVs[C]// Oceans. 2013:1-8.

[13] Yigit H, Yilmaz G. Development of a GPU Accelerated Terrain Referenced UAV Localization and Navigation Algorithm[J]. Journal of Intelligent \& Robotic Systems, 2013, 70(1):477-489.

[14] Zhao L, Gao N, Huang B, et al. A Novel Terrain-Aided Navigation Algorithm Combined With the TERCOM Algorithm and Particle Filter[J]. IEEE Sensors Journal, 2015, 15(2):1124-1131.

[15] B Turan, AT Kutay. Particle Filter Studies on Terrain Referenced Navigation. Position[C], Location and Navigation Symposium (PLANS), IEEE/ION. 2016:949-954

[16] Ş Temel, Ünaldi N, İnce F. Novel terrain relative lunar positioning system using lunar Digital Elevation Maps[C]// International Conference on Recent Advances in Space Technologies. IEEE, 2009:597 - 602.

[17] W. Kang, Z. Long, Research on the TAN fitting for aviation[A].Piezoelectrics and Acoustooptics, 2010,32(5): pp. 754-761.

[18] Eroglu O, Yilmaz G. A novel fast and accurate algorithm for Terrain Referenced UAV localization $[\mathrm{C}] / /$ International Conference on Unmanned Aircraft Systems. 2013:660-667.

[19] Zhen Li. The research on matching algorithn of terrain aided navigation system [D]. Qilu University Of Technology, 2015.

[20] Doucet A,Freitas N,and Gordon N J.Sequential Monte Carlo Methods in Practice.New York:Springer,2001.

[21] SUN Yan-yue,HE Xiao-hai. Image Super-resolution Reconstruction of Wavelet Local-adapt at ion Interpolat ion[J]. Computer Engineering, 2010, 36(13): 183-185.

[22] Yu Hao, Yang Qinke. Comprehensive study of DEM data based on wavelet multiscale analysis [J]. Science of Surveying and Mapping, 2008, 33(3):93-95. 